\title{
SUSTENTABILIDADE AGROPECUÁRIA DE PRODUTORES RURAIS EM MUNICÍPIOS DO SERTÃO DA PARAÍBA
}

\author{
Vicente Maia Pinto Junior \\ Mestre em Sistemas Agroindustriais \\ Universidade Federal de Campina Grande \\ Pombal - Paraíba - Brasil \\ vicente-maia@hotmail.com \\ Ancélio Ricardo de Oliveira Gondim \\ Doutor em Agronomia \\ Universidade Federal de Campina Grande \\ Pombal - Paraíba - Brasil \\ anceliogondim@hotmail.com \\ Patrício Borges Maracajá \\ Doutor Engenheiro Agrônomo \\ Universidade Federal de Campina Grande \\ Pombal - Paraíba - Brasil \\ patriciomaracaja@gmail.com \\ Anderson Bruno Anacleto de Andrade \\ Doutorando em Proteção de Plantas \\ Universidade Federal de Alagoas \\ Maceió - Alagoas - Brasil \\ bdeandrade3@gmail.com
}

\section{RESUMO}

Objetivou-se avaliar o nível de sustentabilidade dos produtores rurais em quatro municípios do Sertão da Paraíba, analisando a contribuição dos indicadores quanto à qualidade de vida, preservação ambiental e capital social. Os dados foram obtidos, mediante aplicação de questionário com 40 produtores rurais de cada município e, com base nestes dados, foram calculados os índices de qualidade de vida, capital social e ambiental, para, então, definir o índice de sustentabilidade. Nos municípios do Sertão paraibano estudados, verificou-se que, segundo os produtores rurais, as atividades pecuárias que apresentam melhor desempenho econômico são a caprinocultura, a apicultura e a bovinocultura. Dentro das atividades agrícolas, destacam-se a fruticultura e a cultura de sequeiro. Em relação ao índice de qualidade de vida, todos os municípios foram caracterizados como nível médio. Para o índice de capital social, apenas Triunfo foi definido como baixo nível, os demais foram classificados como médio. O município de Santa Helena apresentou índice ambiental de médio nível de preservação, sendo as caracterizações dos demais municípios de baixo nível. A sustentabilidade dos produtores rurais, dos municípios de São João do Rio do Peixe, Santa Helena e Poço de José de Moura foi definido como médio. O município de Triunfo foi classificado como de baixo nível de sustentabilidade dos produtores rurais o qual teve contribuição negativa da baixa preservação ambiental e pequena participação social dos produtores.

Palavras-chave: Comportamento ambiental. Desenvolvimento sustentável. Qualidade de vida rural. Semiárido.

\section{AGRICULTURAL SUSTAINABILITY OF RURAL PRODUCERS IN MUNICIPALITIES OF SERTÃO DA PARAÍBA, BRAZIL}

\section{ABSTRACT}

The objective of this study was to evaluate the level of sustainability of rural producers in four municipalities of Sertão da Paraíba, analyzing the contribution of the indicators regarding quality of life, social capital and environmental preservation of producers. The data were obtained through application of questionnaires with 40 rural producers from each municipality and, based on these data, indices of quality life, social and environmental capital were calculated, in order, to calculate the sustainability index. In the municipalities of Sertão, in the state of Paraíba, Brazil, we verified that, according to the rural producers, the livestock activities that present the best economic performance are goat breeding, beekeeping and cattle breeding. Within the agricultural activities, fruit culture and the dry land culture stand out. In relation to the quality of life index, all municipalities were characterized as medium. For the social capital index, only Triunfo was defined as low level, since the others were classified as average. The municipality of Santa Helena presented an environmental index for the rural producers of medium level of preservation, and as characterizations of the other municipalities were low level. The sustainability of rural producers, in the municipalities of São João do Rio do Peixe, Santa Helena and Poço de José de Moura was defined as medium. The municipality of Triunfo was classified as low level of sustainability of the rural producers, which had a negative contribution of the low environmental preservation and small social participation of the producers.

Key words: Environmental behavior. Semi-arid. Sustainable development. Rural quality of life. 


\section{INTRODUÇÃ̃O}

O desenvolvimento sustentável rural baseia-se na premissa do atendimento às necessidades de produção para a população atual, sem comprometer a capacidade das futuras gerações, sendo necessária uma postura efetiva na utilização os recursos naturais, durante as atividades produtivas e na qualidade da dignidade humana (Medeiros, 2015).

$\mathrm{Na}$ busca por opções, que venham a contribuir para o desenvolvimento de práticas sustentáveis, ocorre a abertura para novos nichos de mercados e uma maior diversificação nas suas atividades econômicas rurais (Lima, Castro \& Pires, 2011). Nesse processo de construção de estilos de atividades agropecuárias sustentáveis, nota-se a insustentabilidade dos métodos convencionais e o destaque dos princípios da Agroecologia (Paludo \& CostaBeber, 2012).

Tendo como base a necessidade de produzir e, ao mesmo tempo, preservar o meio ambiente, procurando formas sustentáveis para atender a população, os sistemas de indicadores surgem nesse cenário como uma alternativa para compreensão e minimização das atividades nocivas, e instrumento para a tomada de decisões na produção agropecuária (Sales \& Cândido, 2014).

O desenvolvimento sustentável está fundamentado em três dimensões: a econômica, a social e a ambiental. Assim, torna-se importante a realização de estudos que contemplem as variáveis de ordem organizativa, social e ambiental, onde os aspectos relacionados à qualidade de vida, produção agrícola, educação, saúde, lazer, relações de gênero, associativismo, preservação ambiental, entre outros, se destacam como elementos que apresentam seus reflexos sobre a sustentabilidade na atividade agropecuária (Nascimento, 2012).

Os indicadores de sustentabilidade contribuem com o planejamento e monitoramento para a tomada de decisões em busca de um desenvolvimento sustentável, tendo como principal função o fornecimento de conhecimentos acerca das dimensões da área estudada (socioeconômicas, econômicas, ambientais, institucionais, culturais e entre outras), os quais integram o sistema desenvolvimento sustentável da sociedade (Carvalho, Curi, Carvalho \& Curi, 2011).

Com isso os produtores rurais, que utilizam os indicadores de sustentabilidade para um melhor gerenciamento da sua propriedade integrando as relações sociais, econômicas e ambientais, contribuem de maneira decisiva para o estímulo ao desenvolvimento sustentável no meio rural (Andersson et al., 2017).

A utilização de indicadores é relevante na tomada de decisão e isso é fundamental para realização de ações, tanto por parte de instituições privadas quanto públicas, no fomento à sustentabilidade (Rodrigues \& Rippel, 2015). Nesse sentido, a contemplação de fatores sociais, ambientais e econômicos que possibilita a formação de um perfil claro e coerente da atividade rural, revela-se uma tendência, pois caracteriza os territórios, possibilitando a identificação das alternativas de desenvolvimento. Por este viés, este estudo objetivou avaliar o nível de sustentabilidade dos produtores agropecuários em quatro municípios do Sertão da Paraíba (São João do Rio do Peixe, Santa Helena, Triunfo e Poço Jose de Moura) analisando a contribuição dos indicadores quanto à qualidade de vida, capital social e preservação ambiental.

\section{FUNDAMENTAÇÃO TEÓRICA}

\subsection{Produção rural no Brasil}

A economia brasileira, desde o processo de colonização e crescimento, esteve ligada a vários ciclos agroindustriais. Assim, com a modernização e maior eficiência das técnicas de produção, a atividade rural possui a capacidade de impulsionar outros setores que geram um efeito multiplicador, tanto na economia como no crescimento do Produto Interno Bruto do País (Zambra, Souza \& Pereira, 2015). 
O impacto da participação da agropecuária na economia brasileira também é medido pela expansão doméstica dos setores fornecedores de insumos agrícolas, bem como ao desenvolvimento das agências nos mercados nacionais e internacionais (Baer, 2008).

Um fator importante na atividade rural nacional é a produção familiar, apontando uma participação no empregando, $78,75 \%$ do total da mão de obra no campo (Guanziroli, Buainain, \& Sabbato, 2012). Na região Nordeste, o agronegócio desempenha papel de destaque na economia regional, em que os sistemas com gerenciamento familiar representam cerca de $50 \%$ do agronegócio total (Guilhoto, Azzoni \& Ichihara, 2014).

Os pequenos agricultores ou os agricultores familiares respondem por parcela expressiva da riqueza nacional, mesmo tendo a insuficiência de terras, as dificuldades creditícias, o menor aporte tecnológico, a fragilidade da assistência técnica e a subutilização da mão-de-obra. É considerada a base dos sistemas agroindustriais, por fornecerem matéria-prima para grandes e pequenas empresas, integrando, assim, as cadeias do sistema de agroindústrias (Buainain, 2006; Guilhoto, Silveira, Ichihara \& Azzoni, 2006).

A produção familiar não é somente um fator de desaceleração do fenômeno do êxodo rural e fonte de recursos para famílias de rendas menores, visto que tem, também, importante contribuição para a geração de riquezas no País (Guilhoto, Assumpção, Módolo \& Imori, 2007).

A agricultura familiar, portanto, possibilita um desenvolvimento econômico e social para os que exercem essa ocupação, proporcionando a geração de diversos postos de trabalho, empregos e fluxos de renda, tornando-se, assim, determinante na melhoria da qualidade de vida e fixação do homem no meio rural (Guanziroli, Romeiro, Buainain, Sabbato \& Bittencourt, 2001).

\subsection{Desenvolvimento sustentável rural}

O conceito de desenvolvimento sustentável está relacionado a um processo de construção, em que as várias correntes de pensamento do conhecimento científico estabelecem critérios que orientem as ações de desenvolvimento social e econômico numa perspectiva de simbiose com a natureza (Chaves \& Rodrigues, 2006).

O desenvolvimento sustentável surgiu a partir dos pensamentos sobre a sociedade e a gravidade das dificuldades ambientais, sejam elas naturais, humanas, sociais, laborais ou culturais, visto que as várias questões complexas, que estão presentes nessa temática, são tratadas de forma interdisciplinar, dado que os estudos, o planejamento e as ações acontecem em um contexto abrangente e sistêmico. A sustentabilidade engloba diversas inter-relações entre diferentes grupos sociais e, para cada grupo, questões temporais e espaciais únicas, sendo um procedimento de aprendizado igualitário de extenso prazo, o qual é direcionado por políticas públicas nacionais (Rabelo \& Lima, 2007).

A necessidade do desenvolvimento sustentável rural como projeto político e social tem requerido a orientação de esforços para que possam contribuir como incremento de uma sociedade sustentável, que combata o desperdício, levando em conta o procedimento coletivo e o bem comum, sem violar os direitos individuais de seus membros (Salas-Zapata, Ríos-Osorio \& Castillo, 2011).

O interesse sobre o assunto é crescente, envolvendo, nos últimos tempos, múltiplas questões, tais como, táticas, formas de cultivo limpo, formas de conter a poluição, ecologia industrial, entre diversas adjacências que tendem a apresentar uma diminuição dos efeitos do homem sobre o meio ambiente (Glavic \& Lukman, 2007).

A relevância contemporânea de garantir o desenvolvimento rural sustentável é estipulada, por um lado, pelo crescente atraso econômico e social dos territórios rurais e, por outro, pela sua importância máxima para a nação em questões, tais como, a segurança alimentar, a preservação do solo e recursos ambientais e a utilização de potenciais agrícolas, produtivos e trabalhistas (Erokhin, Heijman \& Ivolga, 2014).

No desenvolvimento sustentável, as estratégias de aproximação do sistema ambiental humano ao nível de sustentabilidade visam a uma harmonização que se perpetue ao longo do tempo.

Revista de Gestão Social e Ambiental - RGSA, São Paulo, v. 12, n. 3, p. 77-94, set./dez. 2018. 
As estratégias intentam a ruptura de paradigmas, por meio de mudanças no entendimento e posicionamento cultural da sociedade (Feil \& Schreiber, 2017).

Nas atividades agropecuárias, o desenvolvimento sustentável deve cumprir alguns objetivos que priorizem a manutenção por longo prazo dos recursos naturais e da produtividade agrícola; o mínimo de impactos adversos ao ambiente; retornos adequados aos produtores; otimização da produção com mínimo de insumos externos; satisfação das necessidades humanas de alimentos e renda; atendimento às necessidades sociais das famílias e das comunidades rurais (Veiga, 1994).

Segundo Pascu (2013), uma alternativa para que desenvolvimento sustentável rural possa ser alcançado é a transição da agricultura de subsistência para a uma atividade orgânica por meio da construção e do fortalecimento de pequenas e médias propriedades nas áreas rurais, possibilitando que os trabalhadores vivam em um ambiente rural de harmonia com a biodiversidade, possibilitando a geração de renda por meio da produção orgânica com maior valor agregado.

O protagonismo social, em estreita relação com as práticas agroecológicas, proporciona avanços no meio rural, visto que possibilita, por meio do empoderamento e da construção da identidade do homem do campo, a busca pelo desenvolvimento rural sustentável (Abreu, Nascimento, Brasil \& Andrade, 2016). A organização dos produtores rurais em associações, no intuito da eficácia técnica e econômica, proporciona ao produtor atuação na produção, beneficiamento e comercialização dos produtos (Silva, Mayorga, Khan \& Lima, 2013).

O fortalecimento das associações e organizadas coletivamente indica um dos princípios para um desenvolvimento rural alternativo à lógica hegemônica. Essas organizações configuram-se como um berço para diversas outras iniciativas dos produtores, possibilitando a obtenção de renda e manutenção no campo com qualidade de vida, rumo a um desenvolvimento rural sustentável (Komarcheski, Sibuya \& Denardin, 2014).

\subsection{Indicadores do desenvolvimento sustentável}

O período de alcance de um ambiente sustentável é considerado uma jornada longa, complexa e dependente do contexto, requerendo flexibilidade para adaptação de projetos aos desafios detectados, com isso, Jiménez, Jawara, Ledeunff, Naylor e Scharp (2017) descrevem a sustentabilidade como um processo iterativo, com base em monitoramento regular para percepção dos principais desafios e desencadeamento de mecanismos para abordá-los em níveis operacionais e estratégicos.

O monitoramento do desenvolvimento rural é um requisito fundamental para a formulação de políticas e planejamentos que auxiliam na compreensão dos processos de desenvolvimento. Os indicadores da sustentabilidade, quando combinados com ferramentas para sua visualização, manipulação e análise, são componentes essenciais do processo de monitoramento (Farrow \& Winograd, 2001).

A utilização dos indicadores proporciona a busca pelo desenvolvimento sob um enfoque integrador, mediante as dimensões sociais, ambientais e econômicas, para a consolidação de uma sociedade sustentável (Kemerich, Ritter \& Borba, 2014). Sendo uma base para medir a sustentabilidade e direcionar, suas políticas visam alcançar uma melhor qualidade de vida. $\mathrm{O}$ sistema de indicadores é considerado como um passo importante do processo de desenvolvimento sustentável, fornecendo informações úteis para as decisões e os planejamentos, além da estratégia de sustentabilidade (Hai et al., 2014).

Os indicadores ou índices de sustentabilidade são ferramentas com propriedades quantitativas que podem identificar quais os aspectos, são os responsáveis e quais devem ser reposicionados ou corrigidos na avaliação do nível sustentável desejável (Feil \& Schreiber, 2017).

Existe uma gama de indicadores de sustentabilidade, contudo Bellen (2004) destaca que, entre os principais sistemas indicadores de sustentabilidade, se consideram as três dimensões elementares da sustentabilidade: a econômica, a social e a ambiental. O ambiental faz referência aos 
recursos naturais; o econômico compreende a renda da propriedade sustentada no tempo; e o fator social referindo-se à igualdade. Porém, determinadas ocasiões admitem que os indicadores necessitam serem desenvolvidos basicamente com base na associação de dados, em que distintos tipos de indicadores podem apresentar-se acentuados em escalas (Gallopin, 1996).

A utilização de indicadores na avaliação, a mensuração, o monitoramento da sustentabilidade é frequente em atividades rurais, na atribuição de índices que auxiliam na identificação pontos críticos ou riscos e os pontos positivos e oportunidades de desenvolvimento. Esses indicadores têm sempre como base o tripé da sustentabilidade e são empregados em todas as regiões do mundo (Ferreira et al., 2012; Michael, Noor \& Figueroa, 2014; Kelly et al., 2018).

As primeiras iniciativas direcionadas a construção de indicadores de desenvolvimento sustentável baseiam-se no documento conhecido como Agenda 21, que representa o consenso determinado pela comunidade internacional sobre o meio ambiente e seus reflexos socioeconômicos e culturais. Em que as diretrizes dessas medidas focam na implementação de planos, programas e projetos vinculados a melhoria da qualidade de vida global, bem como os grandes dilemas da preservação dos recursos para o desenvolvimento sustentável. Dentre as diversas metodologias, apontam-se, os marcos ordenadores de indicadores e as metodologias de construção de índices, em que cada ferramenta tem seu objetivo e deve ser utilizada de acordo com a finalidade do estudo, portanto, podem ser aplicadas de forma isolada ou complementando outra (Rodrigues \& Rippel, 2015).

A primeira dimensão normalmente evidenciada no estudo do desenvolvimento sustentável é a ambiental, estando relacionada ao modelo de produção que deve garantir aos ecossistemas manter sua autorreparação ou capacidade de resiliência (Nascimento, 2012) Os indicadores ambientais relacionados a uma atividade produtiva podem ser adequados e potentes na discussão da sustentabilidade desta atividade, sendo constituídos segundo os princípios de boas práticas e cumprindo a função de apoio às políticas públicas rumo à sustentabilidade (Gomes \& Malheiros, 2012).

Nos indicadores nas dimensões econômicas há composições que não podem ser apenas de ordem relacionada à economia, mas devem buscar constantemente medidas socioeconômicas mais abrangentes, que incluam outras dimensões fundamentais da vida e da condição humana em que a qualidade de vida (ou bem-estar) são mensuráveis pelas evidências relacionadas à economia da felicidade (Alves \& Bastos, 2011; Veiga, 2010).

\section{MÉTODOS}

O trabalho caracteriza-se como uma pesquisa aplicada, que, de acordo com os objetivos, é de característica exploratória com procedimento de levantamento de dados quantitativos e qualitativos de uma amostra, sendo realizada em quatro municípios rurais (Veiga, 2002), localizados no Sertão da Paraíba (Tabela e Figura 1).

Tabela 1. Características gerais dos municípios do Sertão da Paraíba

\begin{tabular}{lccccc}
\multicolumn{1}{c}{ Município } & População & $\begin{array}{c}\text { População } \\
\text { Rural }\end{array}$ & $\begin{array}{c}\text { Área territorial } \\
\mathrm{Km}^{2}\end{array}$ & $\begin{array}{c}\text { Densidade demográfica } \\
\text { hab./km }^{2}\end{array}$ & IDHM \\
\hline São João do Rio do Peixe & 18.201 & 11.316 & 474 & 38,36 & 0,608 \\
Triunfo & 9.220 & 4.911 & 223 & 41,93 & 0,609 \\
Santa Helena & 5.369 & 2.667 & 210 & 25,53 & 0,609 \\
Poço de José de Moura & 3.968 & 2.553 & 100,9 & 39,4 & 0,612 \\
\hline
\end{tabular}

IDHM: Índice de Desenvolvimento Humano

Fonte: Instituto Brasileiro de Geografia e Estatística [IBGE] (2010) 


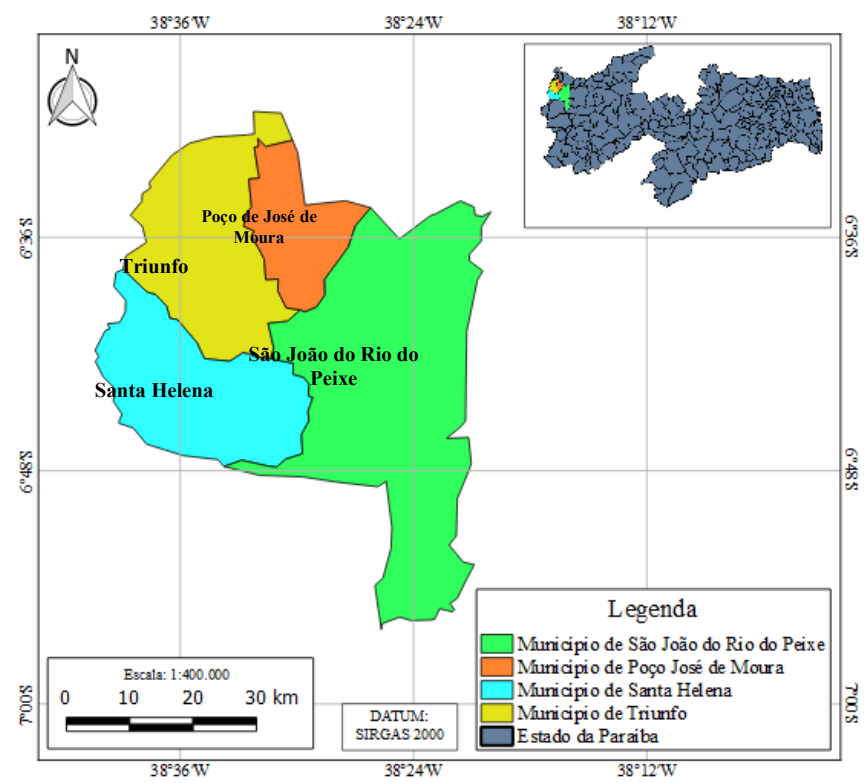

Figura 1. Localização dos municípios de São João do Rio do Peixe, Triunfo, Santa Helena e Poço de José de Moura no Estado da Paraíba. Brasil

Fonte: Autor, 2018

Os dados foram obtidos mediante aplicação de questionários e entrevistas semiestruturados diretamente a 40 produtores rurais em cada município no período entre os meses de julho a setembro de 2016. Os questionários foram adaptados de Khan e Silva (2002) para a determinação do Índice de Sustentabilidade tendo como base indicadores que representam a qualidade de vida, as relações sociais e a preservação ambiental nas atividades rurais.

Os Índices de Qualidade de Vida (IQV), Capital Social (ICS) e Ambiental (IA) foram definidos conforme Khan e Silva (2002), utilizando-se a equação 1 e a contribuição de cada indicador foi obtida de acordo com a equação 2.

$$
\begin{aligned}
& \mathrm{Ic}=\frac{1}{\mathrm{n}} \sum_{\mathrm{j}=1}^{\mathrm{n}}\left(\sum_{\mathrm{i}=1}^{\mathrm{m}} \mathrm{E}_{\mathrm{ij}} \mathrm{P}_{\mathrm{ij}}\right) \cdot\left(\sum_{\mathrm{i}=1}^{\mathrm{m}} \mathrm{E} \max _{\mathrm{i}} \cdot \mathrm{P} \max _{\mathrm{i}}\right)^{-1} \\
& C_{i}=\left(\sum_{i=1}^{n} E_{i j} \cdot P_{i j}\right) \cdot\left[n\left(\sum_{i=1}^{m} E \max _{i} \cdot P \max _{i}\right)\right]^{-1}
\end{aligned}
$$

Em que: Ic: são os índices calculados; Eij: Escore do i-ésimo indicador, alcançado pelo jésimo produtor; Pij: Peso do i-ésimo indicador, alcançado pelo j-ésimo produtor; i:1,..,m (indicadores); $\mathrm{j}: 1, \ldots, \mathrm{n}$ (produtores); Pmaxi: Peso máximo do i-ésimo indicador; Emaxi: Escore máximo do i-ésimo indicador; Ci: Contribuição do indicador (i) no índice dos produtores.

$\mathrm{O}$ IQV foi estabelecido com indicadores relacionados a condições de acesso dos atores à serviços que estão diretamente ligados ao bem-estar (educação, saúde, habitação, aspectos sanitários, lazer e posse de bens duráveis). A comparação entre os valores indicativos, componentes do IQV foi realizada mediante avaliação de indicadores com escore de valor variando de 0 a 3 (Tabela 2). 
Tabela 2. Escores para os indicadores avaliados na composição do Índice de Qualidade de Vida.

\begin{tabular}{|c|c|c|c|c|c|}
\hline \multirow{2}{*}{ Indicadores } & \multicolumn{5}{|c|}{ Escores } \\
\hline & 0 & 1 & 2 & & \\
\hline \multicolumn{6}{|l|}{ 1) Educação } \\
\hline $\begin{array}{l}\text { Escolas na comunidade } \\
\text { 2) Saúde }\end{array}$ & Ausência & Alfabetização & Fundamental 1 & \multicolumn{2}{|c|}{ Fundamental 2} \\
\hline Serviços de saúde & Ausência & Primeiros socorros & Agente de saúde & \multicolumn{2}{|c|}{ Atendimento médico } \\
\hline \multicolumn{6}{|l|}{ 3) Habitação } \\
\hline Construção da residência & Casa de taipa & $\begin{array}{c}\text { Casa de tijolos sem } \\
\text { reboco }\end{array}$ & $\begin{array}{l}\text { Casa de tijolos } \\
\text { com reboco }\end{array}$ & & \\
\hline Tipo de iluminação & Lampião & Energia elétrica & & & \\
\hline \multicolumn{6}{|l|}{ 4) Aspectos sanitários } \\
\hline Tratamento da água & $\begin{array}{l}\text { Nenhum } \\
\text { tratamento }\end{array}$ & $\begin{array}{l}\text { Fervida, filtrada ou } \\
\text { hipoclorito }\end{array}$ & & & \\
\hline $\begin{array}{l}\text { Destino dado aos dejetos } \\
\text { humanos }\end{array}$ & $\begin{array}{l}\text { Céu aberto ou } \\
\text { enterrados }\end{array}$ & Fossa ou esgoto & & & \\
\hline $\begin{array}{l}\text { Destino dado ao lixo } \\
\text { domiciliar } \\
\text { 5) Lazer }\end{array}$ & $\begin{array}{l}\text { Solo ou } \\
\text { queimado }\end{array}$ & Enterrado & $\begin{array}{l}\text { Coleta } \\
\text { Municipal }\end{array}$ & & \\
\hline Estrutura de lazer & Ausência & Campo de futebol & Salão de festas & \multicolumn{2}{|c|}{ Praça pública } \\
\hline 6) Bens duráveis & Ausência & Grupo $1 *$ & Grupos 1 e 2 & \multicolumn{2}{|c|}{ Grupos 1,2 e 3} \\
\hline \multicolumn{6}{|c|}{$\begin{array}{l}\text { *Grupo 1: rádio, ferro de engomar, liquidificador, bicicleta; Grupo 2: máquina de costura, equipamento de som, TV } \\
\text { fogão à gás; geladeira Grupo 3: Celular, computador, motocicleta, carro. }\end{array}$} \\
\hline \multicolumn{6}{|c|}{$\begin{array}{l}\text { O índice de capital social foi estudado com o objetivo de mensurar a participação e o níve } \\
\text { de envolvimento dos produtores nas associações, sindicatos e atividades culturais e políticas em } \\
\text { cada comunidade, atribuindo-lhes valores } 0 \text { (zero) para não participação e } 1 \text { para participação } \\
\text { (Tabela } 3 \text { ). }\end{array}$} \\
\hline \multicolumn{6}{|c|}{ Tabela 3. Escores para os indicadores avaliados na composição do Índice de Capital Socia } \\
\hline \multirow{2}{*}{ Indicadores } & & & & \multicolumn{2}{|c|}{ Escores } \\
\hline & & & & 0 & 1 \\
\hline \multicolumn{4}{|c|}{ 1) Participação ativa nas atividades da associação } & Não & Sim \\
\hline \multicolumn{4}{|c|}{ 2) Apresentação de sugestões nas reuniões } & Não & Sim \\
\hline \multicolumn{4}{|c|}{ 3) Apreciação e aprovação das sugestões em reuniões } & Não & Sim \\
\hline \multicolumn{4}{|c|}{ 4) Decisões da associação aprovadas em reunião } & Não & Sim \\
\hline \multicolumn{4}{|c|}{ 5) Decisões tomadas nas reuniões são executadas pela diretoria } & Não & Sim \\
\hline \multicolumn{4}{|c|}{ 6) Investimentos realizados na associação são submetidos e aprovados nas reuniões } & Não & Sim \\
\hline \multicolumn{4}{|c|}{ 7) Filiação ao sindicato rural } & Não & Sim \\
\hline \multicolumn{4}{|c|}{ 8) Venda de produtos para cooperativa } & Não & Sim \\
\hline \multicolumn{4}{|c|}{ 9) Participação direta na Política Partidária Municipal } & Não & Sim \\
\hline \multicolumn{4}{|c|}{ 10) Participação em atividades culturais na comunidade } & Não & Sim \\
\hline
\end{tabular}

Foram estabelecidos indicadores para a composição do índice ambiental, com base em atividades desenvolvidas pelos produtores que promoveram práticas conservacionistas e que são representativos para a avaliação ambiental e, a estes foram atribuídos valores de 0 a 2 (Tabela 4).

Tabela 4. Escores para os indicadores avaliados na composição do Índice Ambiental

\begin{tabular}{lccc}
\hline \multirow{2}{*}{ Indicadores } & \multicolumn{2}{c}{ Escores } \\
\cline { 2 - 4 } & 0 & 1 & 2 \\
\hline 1) Conservação do solo & Nenhum método & Práticas mecânicas & Práticas Biológicas \\
2) Controle fitossanitário & Agrotóxico & Nenhum método & Biológico \\
3) Uso do fogo & Sim & Não & Sim \\
4) Prática para evitar a degradação do solo & Não & Sim & Sim \\
5) Faz uso de esterco & Não & Não & Begular \\
6) Área de reserva nativa na comunidade & Ruim & & Boa \\
7) Preservação da reserva nativa & & & \\
\hline
\end{tabular}


Para a composição do Índice de Sustentabilidade em particular, foram utilizados os indicadores com base nos índices de Qualidade de Vida, Capital Social e Ambiental, sendo definido conforme a equação 7 .

$$
\mathrm{IS}=\frac{1}{\mathrm{k}} \sum_{\mathrm{h}=1}^{\mathrm{k}} \mathrm{I}_{\mathrm{h}}
$$

Em que: IS: Índice de Sustentabilidade; I: Escore do h-ésimo índice; h: 1,..,k (índices).

A caracterização de todos os índices foi definida em relação a quanto mais próximo de 1 maior o nível de representação na área em estudo. Assim, considerou-se valores de baixo nível os índices entre 0 e 0,49 , médio nível entre 0,50 e 0,79 e índice de elevado nível com valor entre 0,80 e 1,00 (Khan \& Silva, 2002).

\section{RESULTADOS E DISCUSSÃO}

Os produtores rurais de maior participação foram do gênero masculino, em Poço de José de Moura e Santa Helena, corresponderam a 100\% e 95\% da amostra; em São João do Rio do Peixe e Triunfo representaram $68 \%$ e $66 \%$ dos entrevistados. Em relação à residência, aproximadamente, $80 \%$ dos produtores residem na zona rural, sendo uma média geral de todos os municípios. Estudando os aspectos agroeconômicos e sociais dos produtores rurais em comunidade no Agreste paraibano, Farias, Santos, Leitão, Ribeiro e Silva (2016) obtiveram amostra de $76 \%$ dos trabalhadores do gênero masculino, atribuindo esse percentual ao modo de cultivo predominante na comunidade, considerado convencional, em pequenas e médias áreas, que exigia maior esforço braçal dos produtores.

O comportamento da faixa etária dos produtores rurais denota uma distribuição simétrica na faixa de concentração (35 a 50 anos) no município de Santa Helena. Nos demais municípios, verificaram-se desempenhos assimétricos em relação à mediana e uma maior amplitude dos agrupamentos, com faixas de 38 a 55 anos em São João do Rio do Peixe; 40 a 60 anos em Triunfo e 55 a 70 anos em Poço de José de Moura (Figura 2).

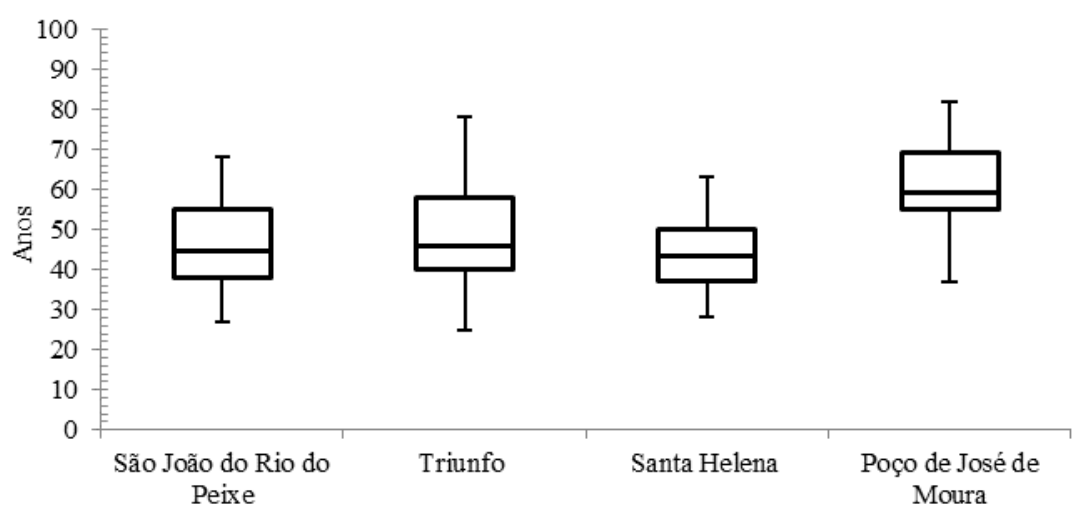

Figura 2. Faixa etária dos produtores rurais nos municípios de São João do Rio do Peixe, Triunfo, Santa Helena e Poço de José de Moura no Sertão da Paraíba

Fonte: Autores, 2018

A idade média dos produtores dos quatros municípios foi de 48 anos. A menor idade foi 25 anos, registrada em Triunfo, e a maior 82 anos, obtida em Poço de José de Moura.

Entre os produtores rurais, o grau de escolaridade apresentado teve como predominância o ensino fundamental em todos os municípios com até $73 \%$ dos entrevistados em Santa Helena (Figura 3). Em pesquisa no também no Alto Sertão paraibano, Vásquez, Barros e Silva (2008), 
caracterizando os produtores da agricultura orgânica de Cajazeiras, observou-se que $67 \%$ da população de amostra tinha como grau de escolaridade apenas o ensino fundamental.

Observa-se, também, que, em Poço de José de Moura e São João do Rio do Peixe, todos os produtores são alfabetizados ou apenas assinam o nome. A formação dos produtores em cursos superiores é registrada nos municipios de Triunfo (5\%), São João do Rio do Peixe (3\%) e em Poço de José de Moura (2,5\%) (Figura 3).

Sugundo Andrade, Santos, Silva e Pimenta (2015), o baixo grau de instrução junto à dificuldade de acesso a informações dificulta aos produtores a identificação dos alertas sobre as práticas nocivas ao meio ambiente e ao ser humano, sendo desprezadas devido ao uso de técnicas culturais transmitidas entre gerações.

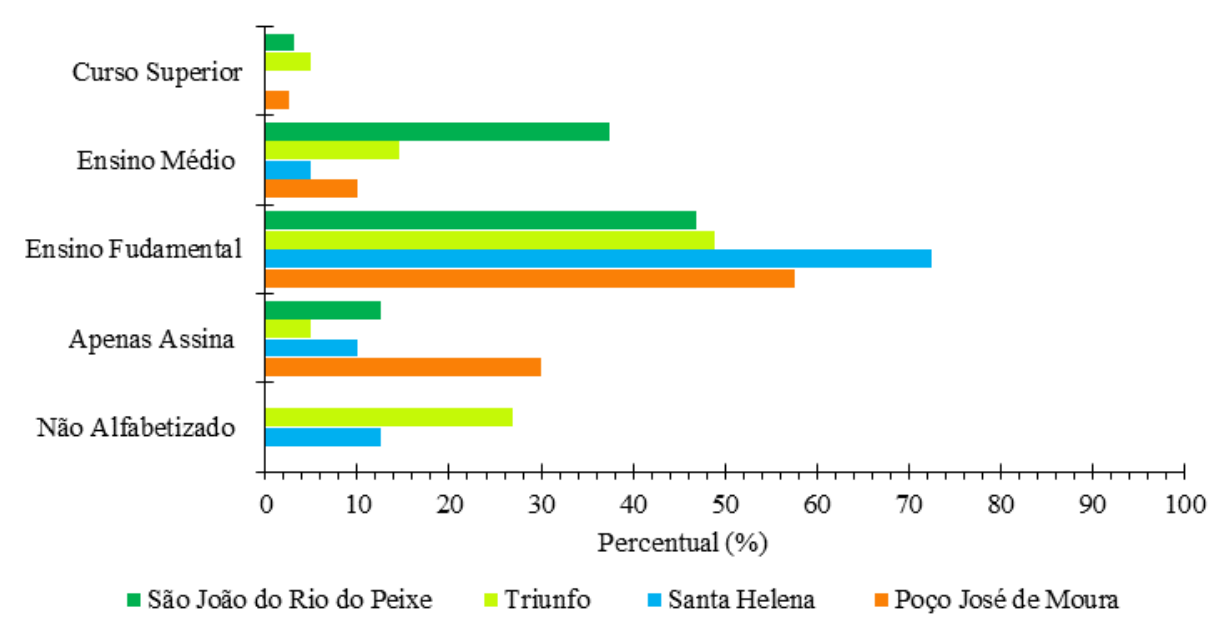

Figura 3. Perfil dos produtores rurais em relação ao grau de escolaridade nos municípios de São João do Rio do Peixe, Triunfo, Santa Helena e Poço de José de Moura no Sertão da Paraíba Fonte: Autores, 2018

O tempo de atuação dos produtores em atividades rurais é demonstrado assimetricamente nas faixas de concentração em todos os municípios estudados com valor médio para a mediana de 35 anos de trabalho. Em Santa Helena, observa-se menor amplitude com faixa de 20 a 30 anos. A idade média dos produtores dos quatros municípios foi de 48 anos. O maior tempo de trabalho foi 72 anos, registrado em Poço de José de Moura e o menor foi de 10 anos observado nos demais municípios (Figura 4).

O tempo de trabalho dos produtores rurais em comunidades no Sertão paraibano está relacionado com a tradição agropecuária das famílias. Sousa (2013), estudando assentamentos no município de Pombal, observou que 93\% dos entrevistados trabalham desde crianças e/ou adolescentes em atividades agropecuárias. Santos e Cândido (2013) observaram que $100 \%$ dos agricultores em uma associação em Lagoa Seca $(\mathrm{PB})$ trabalham há muitos anos na agricultura, e que é uma atividade herdada de seus familiares, assim como é passada, também, para seus filhos. 


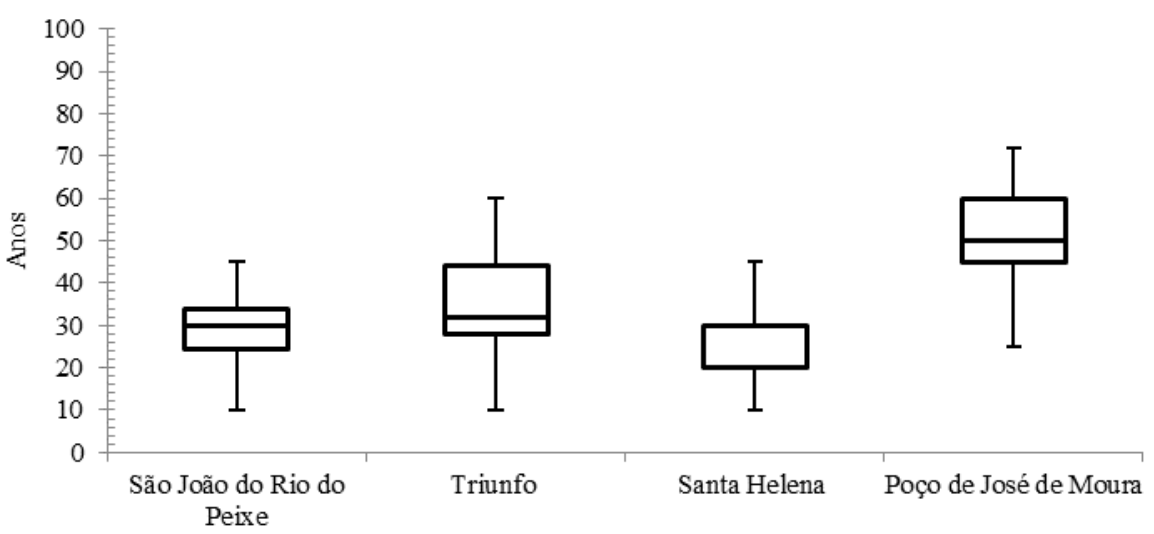

Figura 4. Tmpo de trabalho em atividades agropecuária dos produtores rurais nos municípios de São João do Rio do Peixe, Triunfo, Santa Helena e Poço de José de Moura no Sertão paraibano, 2016

Fonte: Autores, 2018

Em relação à existência de rendas não agrícolas, na maior parte dos municípios, $100 \%$ dos produtores declararam conter fontes que não estão associadas às atividades agropecuárias. Apenas em Triunfo, o percentual daqueles que apresentam rendas não agrícolas foi de $90,2 \%$. As fontes de rendas citadas são: aposentadoria, auxílio do governo federal como programa bolsa-família, vínculo empregatício com as prefeituras municipais e comércios locais.

As rendas não agrícolas para as famílias nordestinas apresentam-se como vetor para a diminuição das desigualdades sociais existentes, possibilitando aos membros familiares absorverem, de forma mais direta, os resultados do crescimento econômico, tendo em vista que as atividades agrícolas da região estão conectadas a uma agricultura familiar pouco diversificada e de baixo poder de agregação (Schneider, 2014).

Nos municípios do sertão paraibano, verificou-se que, segundo os produtores rurais, as atividades pecuárias que apresentam melhor desempenho econômico são a caprinocultura, a apicultura e a bovinocultura. Nas atividades agrícolas, destacam-se a fruticultura e a cultura de sequeiro, sendo esta apenas no município de São João do Rio do Peixe. A indicação das atividades foi constituída a partir da classificação dos produtores em relação ao melhor desempenho econômico, em que foram atribuídos valores de forma crescente em relação à preferência da atividade, ou seja, as que receberam melhor avaliação obtiveram maiores valores (Figura 5).

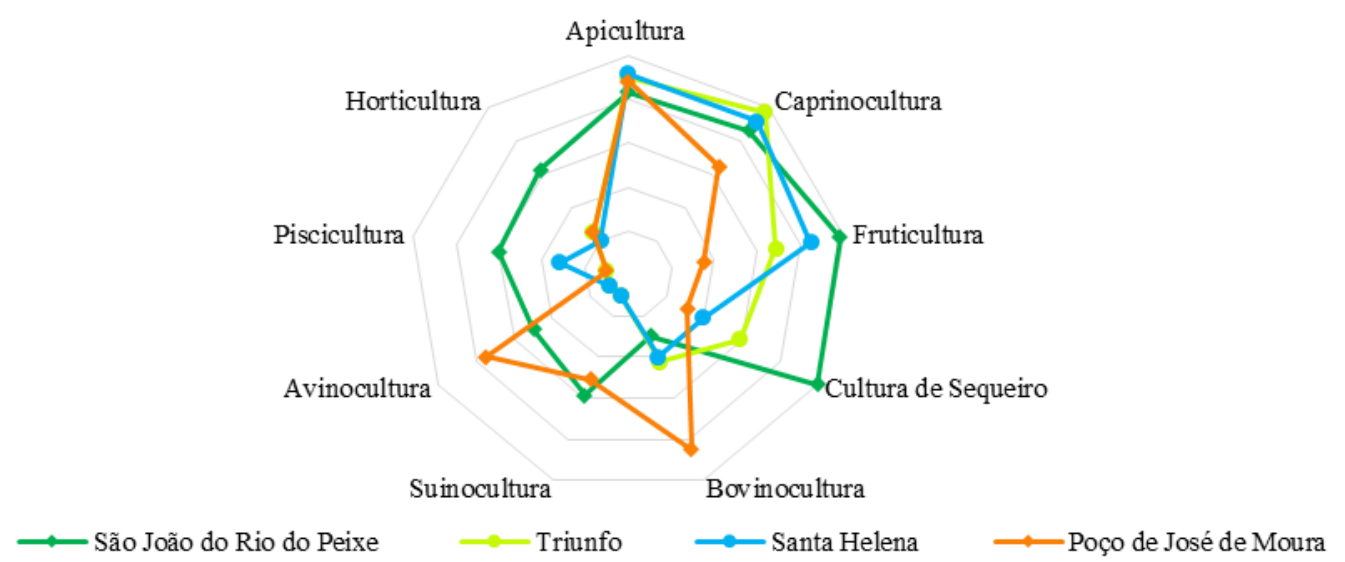

Figura 5. Caracterização das atividades desenvolvidas pelos produtores rurais em relação ao fator econômico nos municípios de São João do Rio do Peixe, Triunfo, Santa Helena e Poço de José de Moura no Sertão da Paraíba. Marcadores mais próximos dos eixos significa melhor desempenho econômico.

Fonte: Autores, 2018 
$\mathrm{Na}$ avaliação do Indice de Qualidade de Vida (IQV), os municípios de São José do Rio do Peixe, Triunfo, Santa Helena, Poço José de Moura obtiveram avaliação considerada como nível médio, conforme disposto na tabela 5. Os indicadores que apresentaram maior participação nos municípios foram saúde, aspectos sanitários, e bens duráveis, sendo os valores percentuais de contribuição de cada indicador, respectivamente, os seguintes: $18 \%, 24 \%$ e $18 \%$ em São João do Rio do Peixe; $21 \%, 15 \%$ e $26 \%$ em Triunfo; $18 \%, 15 \%$ e $22 \%$ em Poço José de Moura; $14 \%$, $28 \%$ e 21\% no município de Santa Helena.

Demonstra, assim, pelo indicador saúde, acesso dos produtores aos agentes comunitários de saúde e atendimento médico. Em relação aos aspectos sanitários, o que se observa de fator positivo é o tratamento dado aos dejetos humanos, por meio de fossa ou esgoto e, de forma negativa, foi o destino do lixo dado por muitos produtores, jogados a céu aberto ou queimado. Para Carneiro et al. (2012), a melhora dos indicadores de saneamento devem ser prioridade para alcançar o desenvolvimento sustentável com qualidade de vida.

$\mathrm{O}$ indicador bens duráveis aponta o poder aquisitivo dos produtores, em que se notou em alguns municípios que todos os entrevistados tinham, pelo menos, dois bens do grupo 3 (Celular, computador, motocicleta, carro). Costa, Silva, Ferreira, Andrade e Costa (2016), avaliando a sustentabilidade dos produtores de hortaliças em comunidades de duas regiões da Paraíba (Agreste e Sertão), também obtiveram como melhores indicadores saúde, aspectos sanitários e bens duráveis, sendo fundamentais para a caracterização do índice como nível médio nas duas comunidades.

Tabela 5. Participação dos indicadores individuais na composição do Índice de Qualidade de Vida dos produtores rurais nos municípios de São João do Rio do Peixe (SJRP), Triunfo (TF), Santa Helena (SH) e Poço de José de Moura (PJM) no Sertão da Paraíba

\begin{tabular}{|c|c|c|c|c|}
\hline Indicadores & SJRP & $\mathrm{TF}$ & $\mathrm{SH}$ & PJM \\
\hline Educação & 0,1332 & 0,0809 & 0,0789 & 0,0921 \\
\hline Saúde & 0,1234 & 0,1271 & 0,1053 & 0,1197 \\
\hline Habitação & 0,1020 & 0,1053 & 0,1053 & 0,1013 \\
\hline Aspectos sanitários & 0,1645 & 0,0911 & 0,2105 & 0,0987 \\
\hline Lazer & 0,0493 & 0,0424 & 0,0842 & 0,1039 \\
\hline Bens duráveis & 0,1250 & 0,1566 & 0,1553 & 0,1487 \\
\hline ÍNDICE DE QUALIDADE DE VIDA (IQV) & 0,6974 & 0,6033 & 0,7395 & 0,6645 \\
\hline
\end{tabular}

Fonte: Autores, 2018

No estado do Ceará, Khan e Silva (2002), avaliando a IQV em assentamentos de reforma agrária, observaram que os indicadores que mais contribuíram para melhor qualidade de vida dos produtores foram aspectos sanitários e bens duráveis corroborando com os resultados dessa pesquisa.

Na composição do Índice de Capital Social (ICS), conforme observa-se na tabela 6, o maior valor foi constatado no município de São João do Rio do Peixe, seguido por Poço José de Moura, Santa Helena, tendo indicador de desenvolvimento em capital social, considerados, de nível médio e Triunfo apresentando um ICS de baixo nível $(0,3439)$.

O indicador que se destaca com maior contribuição para a composição do ICS é a filiação dos produtores ao sindicato rural. Malagodi e Bastos (2003) ressaltam a importância dos sindicatos dos trabalhadores rurais como locus de aglutinação dos agricultores familiares e trabalhadores rurais, vislumbrado como espaço de organização e canal de veiculação dos interesses sindicais e políticos dos trabalhadores rurais.

A venda de produtos para cooperativa foi o indicador de menor contribuição para formação do índice em todos os municípios, mostrando a pouca articulação dos produtores para a comercialização dos produtos de forma coletiva, tendo como principais meios de comércio, a venda direta ou para atravessadores. 
Tabela 6. Participação dos indicadores individuais na composição do Índice de Capital Social dos produtores rurais nos municípios de São João do Rio do Peixe (SJRP), Triunfo (TF), Santa Helena (SH) e Poço de José de Moura (PJM) no Sertão da Paraíba

\begin{tabular}{lcccc}
\multicolumn{1}{c}{ Indicadores } & SJRP & TF & SH & PJM \\
\hline Participação ativa nas atividades com a associação a qual é filiado & 0,0844 & 0,0341 & 0,0600 & 0,1000 \\
Apresentação de sugestões em reunião & 0,0438 & 0,0317 & 0,0575 & 0,0875 \\
Apreciação e aprovação nas reuniões das sugestões apresentadas & 0,0844 & 0,0341 & 0,0575 & 0,0725 \\
Apreciação e aprovação em reuniões das decisões da associação & 0,0844 & 0,0341 & 0,0550 & 0,0725 \\
Execução das decisões tomadas nas reuniões, pela diretoria & 0,0844 & 0,0341 & 0,0575 & 0,0725 \\
Submissão e aprovação dos investimentos da associação & 0,0844 & 0,0390 & 0,0650 & 0,0725 \\
Filiação a sindicato rural & 0,0844 & 0,0659 & 0,0650 & 0,1000 \\
Venda de produtos para cooperativa & 0,0375 & 0,0024 & 0,0150 & 0,0025 \\
Participação na Política Municipal & 0,0375 & 0,0122 & 0,0625 & 0,0125 \\
Participação em atividades culturais & 0,0375 & 0,0561 & 0,0625 & 0,0550 \\
\hline \multicolumn{1}{c}{ ÍNDICE DE CAPITAL SOCIAL (ICS) } & 0,6625 & 0,3439 & 0,5575 & 0,6475 \\
\hline
\end{tabular}

Fonte: Autores, 2018

O capital social possui componentes importantes para compreender o produtor rural enquanto empreendedor (Miyazaki et al., 2004). Para Cheung (2013), a sustentabilidade das produções comandadas por agricultores familiares em comunidades pode ser alcançada com a cooperação presente em todos os elos produtivos, a fim de diminuir os custos de transação. $\mathrm{O}$ capital social é uma importante base facilitadora do processo econômico de desenvolvimento da produção rural, tendo em vista que o sucesso na atividade depende não apenas de crédito, terras, trabalho, acesso a novos mercados e processos tecnológicos, mas também de redes e da organização social enquanto elementos cruciais para o desenvolvimento rural (Bebbinghton, 1999).

$\mathrm{Na}$ tabela 6, observa-se que o município de Santa Helena foi o único que obteve um Índice Ambiental (IA) considerado médio. Esse resultado está associado às práticas de preservação, que contribuíram com um percentual de $22 \%$ para a formação do índice, demonstrando, portanto, que a preservação dos recursos ambientais nas propriedades requer maior atenção por parte dos produtores rurais. Barreto, Khan e Lima (2005), estudando assentamentos rurais em Caucaia no estado do Ceará também observaram maior influência da preservação da reserva nativa na composição do IA.

Os demais municípios são caracterizados como de baixo nível de preservação ambiental. Uma das implicações para esse resultado é a não realização de práticas para evitar a degradação do solo e a não utilização de métodos biológicos para o controle fitossanitário das áreas de cultivo (Tabela 7).

Tabela 7. Participação dos indicadores individuais na composição do Índice Ambiental dos produtores rurais nos municípios de São João do Rio do Peixe (SJRP), Triunfo (TF), Santa Helena (SH) e Poço de José de Moura (PJM) no Sertão da Paraíba

\begin{tabular}{lcccc}
\multicolumn{1}{c}{ Indicadores } & SJRP & TF & SH & PJM \\
\hline É feita conservação do solo & 0,0156 & 0,0732 & 0,1000 & 0,1000 \\
Utiliza método de controle fitossanitário na unidade produtiva & 0,0000 & 0,0366 & 0,1000 & 0,0175 \\
Faz uso do fogo em atividades agropecuárias & 0,0531 & 0,0463 & 0,0050 & 0,0625 \\
Existe prática de plantio para evitar a degradação do solo & 0,0031 & 0,0927 & 0,1000 & 0,0050 \\
Faz uso de esterco & 0,1000 & 0,0390 & 0,0075 & 0,0450 \\
Existe área de reserva nativa na comunidade & 0,0063 & 0,0634 & 0,1000 & 0,0600 \\
Preservação da reserva nativa & 0,0219 & 0,0707 & 0,1175 & 0,1075 \\
\hline \multicolumn{1}{c}{ ÍNDICE AMBIENTAL (IA) } & 0,2000 & 0,4220 & 0,5300 & 0,3975 \\
\hline
\end{tabular}

Fonte: Autores, 2018

No estado do Rio Grande do Norte, Almeida (2005), e no Sertão da Paraíba, Sousa (2013), estudando assentamentos rurais nos municipios de Caraúbas e Pombal, respectivamente, também 
obtiveram comportamento ambiental dos produtores caracterizado como de baixo nível de preservação ambiental, assemelhando-se com os encontrados nessa pesquisa.

A utilização de práticas agroecológicas, tais como uso de estrumes, biofertilizantes, plantio consorciado, rotação de cultura, e a adoção do controle de pragas e/ou doenças com a utilização de plantas defensivas evitando, a pulverização com inseticidas químicos favorecem a sustentabilidade da atividade rural (Santos \& Cândido, 2013).

Na composição do Índice de Sustentabilidade, foram considerados os três índices: IQV, ICS, e IA, tendo em vista representar, da melhor forma, as situações econômicas, sociais e ambientais ocorridas nas comunidades, com suas conquistas e impactos decorrentes (Oliveira, Souza \& Mercante, 2017).

Conforme observa-se na tabela 8 , o indicador que mais contribuiu para a composição do Índice de Sustentabilidade foi o IQV, apresentando-se mais efetivo entre os produtores rurais. Em que os municípios de São João do Rio do Peixe, Santa Helena e Poço José de Moura apresentam IS com maior contribuição da qualidade de vida para os produtores agropecuários. Triunfo foi o que apresentou menor IS, em decorrência da baixa interação social e preservação ambiental dos produtores (Tabela 8).

Tabela 8. Participação dos indicadores na composição do Índice Sustentabilidade dos produtores rurais nos municípios de São João do Rio do Peixe (SJRP), Triunfo (TF), Santa Helena (SH) e Poço de José de Moura (PJM) no Sertão da Paraíba

\begin{tabular}{|c|c|c|c|c|}
\hline Indicadores & SJRP & TF & SH & PJM \\
\hline Qualidade de Vida & 0,6974 & 0,6033 & 0,7395 & 0,6645 \\
\hline Capital social & 0,6625 & 0,3439 & 0,5575 & 0,6475 \\
\hline Ambiental & 0,2000 & 0,3366 & 0,5300 & 0,3975 \\
\hline ÍNDICE DE SUSTENTABILIDADE (IS) & 0,5200 & 0,4564 & 0,6090 & 0,5698 \\
\hline
\end{tabular}

Fonte: Autores, 2018

A sustentabilidade dos produtores agropecuários dos municípios do Sertão da Paraíba, São João do Rio do Peixe, Santa Helena e Poço de José de Moura verifica-se na classificação em nível médio, para o município de Triunfo a caracterização foi de baixo nível de sustentabilidade (Figura 6)



Figura 6. Índice Sustentabilidade dos produtores rurais dos municípios de São João do Rio do Peixe, Triunfo, Santa Helena e Poço de José de Moura no Sertão da Paraíba

Fonte: Autor, 2018 
Utilizando o Índice de Desenvolvimento Sustentável para Municípios (IDSM), Martins e Cândido (2012) verificaram situação de alerta para a sustentabilidade dos municípios paraibanos, tendo como base as dimensões social, demográfica, ambiental, econômica, políticoinstitucional e cultural.

Borges et al. (2014), avaliando a sustentabilidade dos apicultores em municípios do Sertão paraibano, com a participação de São João do Rio do Peixe, Triunfo e Santa Helena, observaram IS caracterizado como de nível alto, e para Poço de José de Moura nível médio, diferenciando dos resultados obtidos neste trabalho com produtores rurais. Esse desempenho pode ser explicado pela diferença entre a amostra avaliada, pois observamos os produtores rurais de forma geral, não apenas os apicultores que participam de associações nas localidades.

\section{CONSIDERAÇÕES FINAIS}

A sustentabilidade dos produtores agropecuários dos municípios, São João do Rio do Peixe, Santa Helena e Poço de José de Moura, é de nível médio, tendo como maior contribuição à qualidade de vida. O município de Triunfo é definido como de baixo nível de sustentabilidade, o qual teve contribuição negativa da baixa preservação ambiental e participação social dos produtores.

Os produtores rurais dos municípios do sertão da Paraíba que, em sua maior parte, residem na zona rural têm acesso as políticas públicas que favorecem a qualidade de vida, com deficiência em relação às práticas de lazer, com ausência de ambientes que proporcione essas atividades nas comunidades rurais.

Nas relações sociais para organização das atividades agropecuárias, os produtores apresentaram boa participação em grupos associativos, porém demostraram baixa influência desses grupos, principalmente na comercialização dos produtos e pouco interesse em questões políticas e culturais dos municípios, assim enfraquecendo as ações do associativismo e/ou cooperativismo entre os produtores.

Os indicadores de preservação ambiental foram os que apresentaram piores resultados, pois os produtores, em sua maioria, realizam atividades pecuárias nas áreas produtivas a partir de métodos não-sustentáveis que agravam os problemas ambientais nessa região e impossibilitam a produção de forma sustentável nas unidades.

Para alcançar uma maior sustentabilidade, políticas extensionistas devem ser realizadas no sentido de orientação dos produtores para ações que preservem o ambiente de produção e maximizem as oportunidades de trabalho em um modelo eficiente e sustentável. A participação social também deve ser estimulada, em grupos que fortaleçam as relações dos produtores com a comercialização, crédito, instruções e incentivo para desenvolvimento das atividades de forma que possibilitem melhor qualidade de vida, desempenho econômico e preservação ambiental.

\section{REFERÊNCIAS}

Abreu, M. K. F., Nascimento, V. S., Brasil, A. A., \& Andrade, D. P. (2016). Protagonismo social e suas contribuições para o desenvolvimento rural sustentável: um estudo de caso no município de Caririaçu - Ceará (Brasil). Sustentabilidade em Debate, 7, 152-168.

Almeida, C. M. (2005). Estudo da sustentabilidade da atividade apícola em duas comunidades do município de Caraúbas - RN. Monografia. Escola Superior de Agricultura de Mossoró. Mossoró, $\mathrm{RN}$, Brasil.

Alves, L. B. \& Bastos, R. P. (2011) Sustentabilidade em Silvânia (GO): o caso dos assentamentos rurais São Sebastião da Garganta e João de Deus. Revista de Economia e Sociologia Rural, 49(2) 419-448. 
Andersson, N. L. M. Noronha, A. P., Ávila, D. T., Costa, J. H. Q. \& Casalinho, H. D. (2017) A multidimensionalidade da sustentabilidade: percepções em um agroecossistema de base familiar característico de assentamentos de reforma agrária. Revista Brasileira de Engenharia e Sustentabilidade, 3(1), 47-57.

Andrade, A. B. A., Santos, J. L. G., Silva, R. A., \& Pimenta, T. A. (2015). Estudo do comportamento dos produtores rurais do município de Livramento - PB sobre os aspectos agroambientais. Anais do Congresso Técnico Científico da Engenharia e da Agronomia, Fortaleza, CE, Brasil, Setembro.

Baer, W. (2008). The Brazilian Economy: Growth and Development (6a ed.). London: Lyanne Rienner Publishers.

Barreto, R. C. S., Khan, A. S., \& Lima, P. V. P. S. (2005). Sustentabilidade dos assentamentos no município de Caucaia-CE. Revista de Economia e Sociologia Rural, 43(2), 225-247.

Bebbinghton, R. (1999). Capitals and capabilities: a framework for analyzing peasant viability, rural livelihoods and powerty. World Development, 27(12), 2021-2044.

Bellen, H. M. V. (2004). Desenvolvimento sustentável: uma descrição das principais ferramentas de avaliação. Ambiente \& Sociologia, 7(1), 67-87.

Borges, M. da G. B., Silva, R. A, Maracajá, P. B., Araújo, A. S., Andrade, A. B. A., \& Cajá, D. F. (2014). Estudo sobre a sustentabilidade: aspectos socioeconômicos e ambientais em cinco associações de apicultores no Sertão da Paraíba. ACTA Apicola Brasilica, 2(2), 1-12.

Buainain, A. M. (2006). Agricultura familiar, agroecologia e desenvolvimento sustentável: questões para debate. (1a ed.). Brasília: Instituto Interamericano de Cooperação para a Agricultura (IICA).

Carneiro, F. F., Franco Netto, G., Corvalan, C., Freitas, C. M. D., \& Sales, L. B. F. (2012). Saúde ambiental e desigualdades: construindo indicadores para o desenvolvimento sustentável. Ciência \& Saúde Coletiva, 17, 1419-1425.

Carvalho, J. R. M. Curi, W. F., Carvalho, E. K. M. A. \& Curi, R. C._(2011) Proposta e validação de indicadores hidroambientais para bacias hidrográficas: estudo de caso na sub-bacia do alto curso do Rio Paraíba, PB. Revista Sociedade e Natureza, 23(2), p.295-310.

Chaves, M. P. S. R. \& Rodrigues, D. C. B. (2006). Desenvolvimento sustentável: limites e perspectivas no debate contemporâneo. Interações, 8(13), 99-106.

Cheung, T. L. (2013). Desenvolvimento da agricultura familiar: investigação sobre o espaço rural e o território como referência para estudar o caso do município de Terenos, MS. Interações, 14(2), 189-195.

Costa, R. O., Silva, R. A., Ferreira, A. C., Andrade, A. B. A., \& Costa, A. da S., Jr. (2016). Qualidade de vida dos produtores de hortaliças em municípios paraibanos. Anais do Congresso Técnico Científico da Engenharia e da Agronomia, Foz do Iguaçu, PR, Brasil, agosto.

Erokhin, V., Heijman, W., \& Ivolga, A. (2014). Sustainable rural development in Russia through diversification: the case of the Stavropol region. Visegrad Journal on Bioeconomy and Sustainable Development, 3(1), 20-25. 
Farias, J. A., Santos, J. J. F., Leitão, E. T. C., Ribeiro, M. D. S., \& Silva, A. J. (2016). Aspectos agroeconômicos e sociais dos produtores rurais de hortaliças de uma região do Agreste paraibano. Anais do Congresso Técnico Científico da Engenharia e da Agronomia, Foz do Iguaçu, PR, Brasil, agosto.

Farrow, A. \& Winograd, M. (2001). Land use modelling at the regional scale: An input to rural sustainability indicators for Central America. Agriculture, Ecosystems and Environment, 85(1), 249-268.

Feil, A. A. \& Schreiber, D. (2017). Sustentabilidade e desenvolvimento sustentável: desvendando as sobreposições e alcances de seus significados. Cadernos Ebape.BR, 14(3), 667-681.

Ferreira, J. M. L., Viana, J. H. M., Costa, A. M., Sousa, D. V. \& Fontes, A. A. (2012) Indicadores de Sustentabilidade em Agroecossistemas. Informe Agropecuário, 33(271) 12-25.

Gallopin, G. C. (1996). Environmental and sustainability indicators and the concept of situational indicators. A system approach. Environmental Modelling \& Assessment, 1(3), 101-117.

Glavic, P. \& Lukman, R. (2007). Review of sustainability terms and their definitions. Journal of Cleaner Production, 15(18), 1875-1885.

Gomes, P. R., \& Malheiros, T. F. (2012). Proposta de análise de indicadores ambientais para apoio na discussão da sustentabilidade. Revista Brasileira de Gestão e Desenvolvimento Regional, 8(2), 151-169.

Guanziroli, C. E., Buainain, A. M. \& Sabbato, A. D. (2012) Dez anos de evolução da agricultura familiar no Brasil: (1996 e 2006). Revista de Economia e Sociologia Rural, 50(2), 351-370.

Guanziroli, C., Romeiro, A., Buainain, A. N., Sabbato, A. D., \& Bittencourt, G. (2001) Agricultura Familiar e Reforma Agrária no Século XXI. (1a ed.). Rio de Janeiro: Garamond.

Guilhoto, J. J. M., Assumpção M., Módolo, D. B., \& Imori, D. (2007, julho). O PIB do Agronegócio no Brasil e no Estado da Bahia. Anais do Congresso da Sociedade Brasileira de Economia e Sociologia Rural, Londrina, PR, Brasil.

Guilhoto, J. J. M., Azzoni, C. R., \& Ichihara, S. M. (2014). Contribuição da agricultura e do agronegócio familiar para o PIB do Nordeste. Revista Econômica do Nordeste, 45, 157-174.

Guilhoto, J. J. M., Silveira, F. G., Ichihara, S. M., \& Azzoni, C. R. (2006). A importância do agronegócio familiar no Brasil. Revista de Economia e Sociologia Rural, 44(3), 355-382.

Hai, L. T., Hai, P. H., Ha, P. T. T., Ha, N. M., Dai, L. T., Hoa, P. V., Huan, N. C., \& Cam, L. V. (2014). A system of sustainability indicators for the province of thai binh, Vietnam. Social Indicators Research, 116(3), 661-679.

Instituto Brasileiro de Geografia e Estatística (2010). Censo demográfico. Recuperado em 20 novembro, 2016, de: http://cod.ibge.gov.br/3S1.

Jiménez, A., Jawara, D., Ledeunff, H., Naylor, K. A., \& Scharp, C. (2017). Sustainability in practice: experiences from rural water and sanitation services in West Africa. Sustainability, 9(3), 114. 
Kelly, E., Latruffe, L., Desjeux, Y. Ryan, M., Uthes, S., Diazabakana A., Dillon, E. \& Finn, J.(2018) Sustainability indicators for improved assessment of the effects of agricultural policy across the EU: Is Fadn the answer? Ecological Indicators, 89, 903-911.

Kemerich, P. D. C., Ritter, L. G., \& Borba, W. F. (2014). Indicadores de sustentabilidade ambiental: métodos e aplicações. Remoa, 13(5), 3723-3736.

Khan, A. S. \& Silva, A. T. B. (2002). Reforma agrária solidária, assistência técnica e desenvolvimento rural no Estado do Ceará. Revista Econômica do Nordeste, 33(3), 593-614.

Komarcheski, R., Sibuya, N., \& Denardin, V. F. (2014). O associativismo em Guaraqueçaba -PR como mecanismo de desenvolvimento rural sustentável. Cadernos de Agroecologia, 9(1), 01-06.

Lima, F. A. X., Castro, M. F., \& Pires, M. L. L. S. (2011). Turismo rural: experiências no Agreste e Sertão de Pernambuco. Rosas dos Ventos, 3(2), 172-181.

Malagodi, E. \& Bastos, V. S. (2003). Sindicato de trabalhadores rurais e agricultura familiar. Anais do Congresso Brasileiro de Sociologia. Campinas, SP, Brasil.

Martins, M. F. \& Cândido, G. A. (2012) Índices de desenvolvimento sustentável para localidades: uma proposta metodológica de construção e análise. Revista de Gestão Social e Ambiental, 6(1), 0319.

Medeiros, K. N. (2015). Desenvolvimento rural e agricultura familiar em áreas de intervenção estatal: o caso do assentamento Eldorado dos Carajás II (RN). Dissertação de mestrado, Universidade Federal Rural do Semiárido, Mossoró, RN, Brasil.

Michael, F. L., Noor, Z. Z., \& Figueroa, M. J. (2014). Review of urban sustainability indicators assessment-case study between Asian countries. Habitat International, 44, 491-500.

Miyazaki, J., Teodoro, P. A. V. B, Raizel, T., Gaffuri, J., Scmidt, R. M., Nazzari, K. R., Bertolini, G. R. F., \& Nazarri, M. T. (2004). Capital social e empreendedorismo rural: a agricultura familiar no oeste do Paraná. Anais do Seminário do centro de ciências sociais aplicadas de cascavel. Cascavel, PR, Brasil.

Nascimento, E. P. (2012) Trajetória da sustentabilidade: do ambiental ao social, do social ao econômico. Estudos Avançados, 26(74), 51-64.

Nascimento, L. F. (2012). Gestão ambiental e sustentabilidade. (1a. ed.) Florianópolis: Universidade Federal de Santa Catarina.

Oliveira, R. D., Souza, C. C., Mercante, M. A. (2017) Análise e diagnóstico da sustentabilidade do assentamento rural Eldorado II, no município de Sidrolândia (MS). IGepec, 21(1), 149-168.

Paludo, R. \& CostaBeber, J. A. (2012) Sistemas agroflorestais como estratégia de desenvolvimento rural em diferentes biomas brasileiros. Revista Brasileira de Agroecologia, 7(2), 63-76.

Pascu, A. D. (2013). Evolution of organic agriculture in Romania and its importance in sustainable rural development. Management, Economic Engineering in Agriculture and Rural Development, 13(2), 313-318. 
Rabelo, L. R. \& Lima, P. V. P. S. (2007). Indicadores de sustentabilidade: uma sequência metodológica para a mensuração do processo ao desenvolvimento sustentável. Revista Eletrônica do Prodema, 1(1), 55-76.

Rodrigues, K. F., \& Rippel, R. (2015). Desenvolvimento sustentável e técnicas de mensuração. Revista de Gestão Ambiental e Sustentabilidade, 4(3), 73-88.

Salas-Zapata, W., Ríos-Osorio, L., \& Castillo, J. A. D. (2011). La ciencia emergente de la sustentabilidad: de la práctica científica hac ia la constitución de una ciencia. Interciência, 36(9), 699-706.

Sales, R. M. M. \& Cândido, G. A. (2014) Aplicação do sistema de indicadores do desenvolvimento alternativo e sustentável na agricultura familiar de Várzea Comprida dos Oliveiras - Pombal/PB. Revista Verde de Agroecologia e Desenvolvimento Sustentável, 9(2), 144-161.

Santos, J. G. \& Cândido, G. A. (2013) Sustentabilidade e agricultura familiar: um estudo de caso em uma associação de agricultores rurais. Revista de Gestão Social e Ambiental, 7(1), 70-86. Schneider, S. (2014). Evolução e Características da Agricultura Familiar no Brasil. Revista Alasru, 9, 21-52.

Silva, E. N., Mayorga, R. D., Khan, A. S., \& Lima, P. V. P. S. (2013). Análise da comercialização e do associativismo apícola dos municípios de Tabuleiro do Norte e Limoeiro do Norte: Um estudo de caso. Enciclopédia Biosfera, 9(16), 85-105.

Sousa, L. C. F. S. (2013) Sustentabilidade da apicultura: aspectos socioeconômicos e ambientais em assentamentos rurais no semiárido paraibano. Dissertação de Mestrado, Universidade Federal de Campina Grande, Pombal, PB, Brasil.

Vásquez, S. F., Barros, J. D. S., \& Silva, M. F. P. (2008) Agricultura orgânica: caracterização do seu produtor na cidade de Cajazeiras-PB. Revista Verde de Agroecologia e Desenvolvimento Sustentável, 3(2), 87-97.

Veiga, J. E. (1994). Problemas da transição à agricultura sustentável. Estudos econômicos, 24, 9-29.

Veiga, J. E. (2010) Indicadores de sustentabilidade. Estudos Avançados, 24(68), 39-52.

Veiga, J. E. da. (2002). Cidades Imaginárias: o Brasil é menos urbano do que se calcula. Campinas: Editores Associados.

Zambra, E. M., Souza, P. A. R., \& Pereira, R. S. (2015). Os impactos da produção de soja e a dinâmica do desenvolvimento em sorriso MT. Pretexto, 16(3), 92-105.

Data da submissão: 17/01/2018

Data de aceite: $08 / 03 / 2018$ 